\title{
Chandra and HESS observations of the supernova remnant CTB 37B
}

F. Aharonian ${ }^{1,13}$, A. G. Akhperjanian ${ }^{2}$, U. Barres de Almeida ${ }^{8, \star}$, A. R. Bazer-Bachi ${ }^{3}$, B. Behera ${ }^{14}$, M. Beilicke ${ }^{4}$, W. Benbow ${ }^{1}$, K. Bernlöhr ${ }^{1,5}$, C. Boisson ${ }^{6}$, V. Borrel ${ }^{3}$, I. Braun ${ }^{1}$, E. Brion ${ }^{7}$, J. Brucker ${ }^{16}$, R. Bühler ${ }^{1}$, T. Bulik ${ }^{24}$, I. Büsching ${ }^{9}$, T. Boutelier ${ }^{17}$, S. Carrigan ${ }^{1}$, P. M. Chadwick ${ }^{8}$, R. C. G. Chaves ${ }^{1}$, L.-M. Chounet $^{10}$, A. C. Clapson ${ }^{1}$, G. Coignet ${ }^{11}$, R. Cornils ${ }^{4}$, L. Costamante ${ }^{1,28}$, M. Dalton ${ }^{5}$, B. Degrange ${ }^{10}$, H. J. Dickinson ${ }^{8}$, A. Djannati-Ataii ${ }^{12}$, W. Domainko ${ }^{1}$, L. O’C. Drury ${ }^{13}$, F. Dubois ${ }^{11}$, G. Dubus ${ }^{17}$, J. Dyks ${ }^{24}$, K. Egberts ${ }^{1}$, D. Emmanoulopoulos ${ }^{14}$, P. Espigat ${ }^{12}$, C. Farnier ${ }^{15}$, F. Feinstein ${ }^{15}$, A. Fiasson ${ }^{15}$, A. Förster ${ }^{1}$, G. Fontaine ${ }^{10}$, S. Funk ${ }^{30}$, M. Füßling ${ }^{5}$, S. Gabici ${ }^{13}$, Y. A. Gallant ${ }^{15}$, B. Giebels ${ }^{10}$, J. F. Glicenstein ${ }^{7}$, B. Glück ${ }^{16}$, P. Goret ${ }^{7}$, C. Hadjichristidis ${ }^{8}$, D. Hauser ${ }^{14}$, M. Hauser ${ }^{14}$, G. Heinzelmann ${ }^{4}$, G. Henri ${ }^{17}$, G. Hermann ${ }^{1}$, J. A. Hinton ${ }^{25}$, A. Hoffmann ${ }^{18}$, W. Hofmann ${ }^{1}$, M. Holleran ${ }^{9}$, S. Hoppe ${ }^{1}$, D. Horns ${ }^{4}$, A. Jacholkowska ${ }^{15}$, O. C. de Jager ${ }^{9}$, I. Jung ${ }^{16}$, K. Katarzyński ${ }^{27}$, S. Kaufmann ${ }^{14}$, E. Kendziorra ${ }^{18}$, M. Kerschhaggl ${ }^{5}$, D. Khangulyan ${ }^{1}$, B. Khélifi ${ }^{10}$, D. Keogh ${ }^{8}$, Nu. Komin ${ }^{15}$, K. Kosack ${ }^{1}$, G. Lamanna ${ }^{11}$, I. J. Latham ${ }^{8}$, M. Lemoine-Goumard ${ }^{31}$, J.-P. Lenain ${ }^{6}$, T. Lohse ${ }^{5}$, J. M. Martin ${ }^{6}$, O. Martineau-Huynh ${ }^{19}$, A. Marcowith ${ }^{15}$,

C. Masterson ${ }^{13}$, D. Maurin ${ }^{19}$, T. J. L. McComb ${ }^{8}$, R. Moderskii ${ }^{24}$, E. Moulin ${ }^{7}$, M. Naumann-Godo ${ }^{10}$, M. de Naurois ${ }^{19}$, D. Nedbal ${ }^{20}$, D. Nekrassov ${ }^{1}$, S. J. Nolan ${ }^{8}$, S. Ohm ${ }^{1}$, J.-P. Olive ${ }^{3}$, E. de Oña Wilhelmi ${ }^{12}$, K. J. Orford ${ }^{8}$, J. L. Osborne ${ }^{8}$, M. Ostrowski ${ }^{23}$, M. Panter ${ }^{1}$, G. Pedaletti ${ }^{14}$, G. Pelletier ${ }^{17}$, P.-O. Petrucci ${ }^{17}$, S. Pita ${ }^{12}$, G. Pühlhofer $^{14}$, M. Punch ${ }^{12}$, A. Quirrenbach ${ }^{14}$, B. C. Raubenheimer ${ }^{9}$, M. Raue ${ }^{1}$, S. M. Rayner ${ }^{8}$, M. Renaud ${ }^{1}$, F. Rieger ${ }^{1}$, O. Reimer ${ }^{29}$, J. Ripken $^{4}$, L. Rob ${ }^{20}$, S. Rosier-Lees ${ }^{11}$, G. Rowell ${ }^{26}$, B. Rudak ${ }^{24}$, J. Ruppel ${ }^{21}$, V. Sahakian ${ }^{2}$, A. Santangelo ${ }^{18}$, R. Schlickeiser ${ }^{21}$, F. M. Schöck ${ }^{16}$, R. Schröder ${ }^{21}$, U. Schwanke ${ }^{5}$, S. Schwarzburg ${ }^{18}$, S. Schwemmer ${ }^{14}$, A. Shalchi ${ }^{21}$, J. L. Skilton ${ }^{25}$, H. Sol ${ }^{6}$, D. Spangler ${ }^{8}$, Ł. Stawarz ${ }^{23}$, R. Steenkamp ${ }^{22}$, C. Stegmann ${ }^{16}$, G. Superina ${ }^{10}$, P. H. Tam ${ }^{14}$, J.-P. Tavernet ${ }^{19}$, R. Terrier ${ }^{12}$, C. van Eldik ${ }^{1}$, G. Vasileiadis ${ }^{15}$, C. Venter ${ }^{9}$, J. P. Vialle ${ }^{11}$, P. Vincent ${ }^{19}$, M. Vivier ${ }^{7}$, H. J. Völk ${ }^{1}$, F. Volpe ${ }^{10,28}$, S. J. Wagner ${ }^{14}$, M. Ward ${ }^{8}$, A. A. Zdziarski ${ }^{24}$, and A. Zech ${ }^{6}$

(Affiliations can be found after the references)

Received 25 February 2008 / Accepted 11 May 2008

\section{ABSTRACT}

We discovered the $>100 \mathrm{GeV} \gamma$-ray source, HESS J1713-381, apparently associated with the shell-type supernova remnant (SNR) CTB 37B, using HESS in 2006. In 2007 we performed X-ray follow-up observations with Chandra with the aim of identifying a synchrotron counterpart to the $\mathrm{TeV}$ source and/or thermal emission from the SNR shell. These new Chandra data, together with additional TeV data, allow us to investigate the nature of this object in much greater detail than was previously possible. The new X-ray data reveal thermal emission from a $\sim 4^{\prime}$ region in close proximity to the radio shell of CTB 37B. The temperature of this emission implies an age for the remnant of $\sim 5000$ years and an ambient gas density of $\sim 0.5 \mathrm{~cm}^{-3}$. Both these estimates are considerably uncertain due to the asymmetry of the SNR and possible modifications of the kinematics due to efficient cosmic ray $(\mathrm{CR})$ acceleration. A bright $\left(\approx 7 \times 10^{-13} \mathrm{erg} \mathrm{cm}^{-2} \mathrm{~s}^{-1}\right)$ and unresolved $\left(<1^{\prime \prime}\right)$ source (CXOU J171405.7-381031), with a soft $(\Gamma \approx 3.3)$ non-thermal spectrum is also detected in coincidence with the radio shell. Absorption indicates a column density consistent with the thermal emission from the shell, suggesting a genuine association rather than a chance alignment. The observed TeV morphology is consistent with an origin in the complete shell of CTB 37B. The lack of diffuse non-thermal X-ray emission suggests an origin of the $\gamma$-ray emission via the decay of neutral pions produced in interactions of protons and nuclei, rather than inverse Compton (IC) emission from relativistic electrons.

Key words. ISM: supernova remnants - gamma rays: observations - X-rays: individuals: G348.7+0.3

\section{Introduction}

Approximately 50 sources of very-high-energy (VHE; $>100 \mathrm{GeV}$ ) $\gamma$-rays have now been detected along the plane of our Galaxy (Aharonian et al. 2006b; Hinton 2007). Some of these sources are (at least partially) coincident with supernova remnants (SNRs), consistent with the paradigm of cosmic-ray acceleration at SNR shocks. Resolved VHE $\gamma$-ray emission from the shells of two nearby $(d \sim 1 \mathrm{kpc})$ SNRs has been detected from: RX J0852.0-4622 (Aharonian et al. 2005) and

^ Supported by CAPES Foundation, Ministry of Education of Brazil.
RX J1713.7-3946 (Aharonian et al. 2007). However, in more distant objects emission from the shell is often not resolvable with current instruments and there is ambiguity in the origin of the VHE emission. In several cases, pulsar wind nebulae (PWNe; for a review see Gaensler \& Slane 2006), powered by neutron stars left behind in the supernova responsible for the remnants, are viable alternative acceleration sites (for example, in the cases of HESS J1813-178, Funk et al. 2007a; and HESS J1804-216, Aharonian et al. 2006b).

The sensitivity to detect much more distant $\gamma$-ray SNRs $(d \gg 1 \mathrm{kpc})$, and hence learn more about the population of such objects, has been achieved only in a few regions of our 
galaxy where deep observations have taken place with the most sensitive available $\mathrm{TeV}$ instruments. One such region is that within a few degrees of RX J1713.7-3946, where a $>50 \mathrm{~h}$ observation with the 4-telescope HESS system results in a pointsource sensitivity of $1.5 \times 10^{-13}$ photons cm $\mathrm{cm}^{-2} \mathrm{~s}^{-1}$ above $1 \mathrm{TeV}$ (or $\sim 0.7 \%$ of the flux of the Crab Nebula). Within this region $\left(l \sim 348^{\circ}, b \sim 0^{\circ}\right)$, a $\gamma$-ray source, HESS J1713-381 was detected in coincidence with the CTB 37 region of radio emission (Aharonian et al. 2006b). This region consists of three supernova remnants, CTB 37A (which consists of two separate remnants overlapping in projection: G348.5+0.1 and G348.5+0.0) and CTB 37B (G 348.7+0.3; Kassim et al. 1991). The first deep TeV measurements resulted in a significant excess close to CTB 37B and a less significant excess close to CTB 37A (Aharonian et al. 2006b). Including the more recent HESS data discussed here, the latter source (HESS J1714-385) is detected with a high level of confidence and is discussed in detail in a separate paper (Aharonian et al. 2008).

At radio wavelengths, CTB 37A and B have very similar properties (Clark \& Stephenson 1975): similar surface brightnesses $\left(\sim 0.2 \mathrm{Jy} \operatorname{arcsec}^{-2}\right)$, spectral indices $(\alpha \sim 0.3$, Kassim et al. 1991) and sizes $\left(\sim 5^{\prime}\right)$. CTB 37A is thought to be interacting with surrounding molecular clouds (with densities of 100-1000 $\mathrm{cm}^{-3}$; Reynoso \& Mangum 2000) and appears to have significant $\mathrm{OH}$ maser emission, indicative of dense, shockheated gas (Frail et al. 1996). No maser emission has been confirmed from the direction of CTB 37B.

The distance to CTB 37B has been estimated by a number of authors. Caswell et al. (1975) used HI absorption measurements to determine the kinematic distance to CTB 37B (using the rotation curve of Schmidt \& Blaauw 1965) to be $10.2 \pm 3.5 \mathrm{kpc}$. A similar result was found for the distance to CTB 37A leading to the claim that CTB 37A and B are close not only in projection, but also along the line of sight. Reinterpreting this result using a more recent galactic rotation curve model (Brand \& Blitz 1993 ) yields a distance range of 5-9 kpc (we note that the value adopted in the catalogue of Green 2006, is $8 \mathrm{kpc}$ ).

The age of CTB 37B is also uncertain. Clark \& Stephenson (1975) give age estimates for both CTB 37A and B of $\sim 1500$ years and claim that there is a possibility that either source could be the remnant of the supernova of AD 393. This claim is further investigated by Downes (1984) who concluded that although such an association is possible, the remnants may in fact be considerably older.

Both to improve our understanding of the SNR CTB 37B and to investigate the nature of HESS J1713-381, sensitive X-ray measurements of this region were considered highly desirable. We observed the field of CTB 37B with Chandra in early 2007. These new X-ray data, together with additional HESS data, are discussed here, with the aim of elucidating the relationship between these two objects.

\section{HESS observations and analysis}

We observed the region toward CTB 37 between April and October 2004 using the High Energy Stereoscopic System (HESS) during a survey of the Galactic Plane, and in pointed observations toward the SNR RX J1713.7-3946, yielding $37 \mathrm{~h}$ of on-source live-time (Aharonian et al. 2006b). We detected VHE gamma-ray emission at a $6.3 \sigma$ significance level from a region of rms size $0.06^{\circ} \pm 0.04^{\circ}$, spatially coincident with the SNR complex, at a position $\mathrm{RA}=17^{\mathrm{h}} 13^{\mathrm{m}} 57.6^{\mathrm{s}}, \mathrm{Dec}=38^{\circ} 12^{\prime} 0^{\prime \prime}$ (epoch J2000) and was announced as a new source HESS J1713-381. Initial spectral analysis of this source led to a photon index of $2.27 \pm 0.48$ and a flux above $200 \mathrm{GeV}$ of $(4.2 \pm 1.5) \times$ $10^{-12} \mathrm{~cm}^{-2} \mathrm{~s}^{-1}$. The region has since been re-observed with HESS and the data set used for analysis here includes all telescope pointings within $1.8^{\circ}$ from the best fit position of the source, leading to a total on-source live-time of $55.4 \mathrm{~h}$. Correcting this to the equivalent on-axis exposure yields $40.8 \mathrm{~h}$. The observations were performed over a large range of zenith angles $\left(14^{\circ}-60^{\circ}\right)$ with an average value of $35^{\circ}$.

We carried out the analysis using standard HESS procedures (for details, see Aharonian et al. 2006a). An independent model analysis (de Naurois 2006) was performed and yielded consistent results. Hard cuts (image size cut 200 photoelectrons (p.e.)) provide a higher signal to noise ratio and a better PSF for 2-D images at the expense of an increased energy threshold and were applied for morphological analysis. Standard cuts ( 80 p.e.) provide a wider energy range and were thus applied for spectral extraction. The average energy threshold of the data set was $370 \mathrm{GeV}$ for hard cuts and $250 \mathrm{GeV}$ for standard cuts.

Two different background estimation models were used in the analysis of the data (described in detail in Berge et al. 2007). For spectral analysis, a reflected-region background model was applied using only off-regions with angular displacement less than $1.8^{\circ}$ from the best fit source position in an attempt to reduce systematic errors arising from the strong exposure gradient across the field of view (due to most pointed observations being directed at RX J1713.7-3946). For morphological analysis and 2-D image generation, used for determining the best fit position of the source, a ring background model was used with ring radius $0.5^{\circ}$, excluding all known $\gamma$-ray sources such as HESS J1714-385 from the background region.

The integration region used for estimating the flux and differential energy spectrum of the VHE emission was a circle of radius $0.187^{\circ}$ centred on the best fit position. This size was chosen so as to maximise the encompassed excess whilst minimising contamination from HESS J1714-385. From the source-fitting it was estimated that this region contained $93 \%$ of the total emission from HESS J1713-381 and included an estimated $11 \%$ contamination from HESS J1714-385.

Figure 1 shows a smoothed, background subtracted and an acceptance corrected image of the region surrounding CTB 37. $\mathrm{TeV}$ emission is observed from both HESS J1713-381 (the centroid of which is marked with a filled black triangle) and HESS J1714-385. Radio contours, taken from the Molonglo Galactic Plane Survey at $843 \mathrm{MHz}$ (Green et al. 1999), show emission only from the eastern side of the SNR CTB 37B as well as a faint plateau of emission extending to the south-east of the remnant. A circle of radius 5.1' has been drawn onto the image in Fig. 1 to represent the size of the SNR based on the partial radio shell. The centroid of the TeV emission from HESS J1713-381 is located at $\mathrm{RA}=17^{\mathrm{h}} 13^{\mathrm{m}} 54^{\mathrm{s}} \pm 4_{\text {stat }}^{\mathrm{s}}$, Dec $=-38^{\circ} 11^{\prime} 58^{\prime \prime} \pm 45^{\prime \prime}$ stat, close to the centre of this proposed shell. The systematic error in the source location is estimated to be $20^{\prime \prime}$ in both coordinates. The source shows an excess of $292 \pm 36$ counts with a statistical significance (using the likelihood method of Li \& Ma 1983) of $8.6 \sigma$. A somewhat higher significance is obtained using the model analysis (Aharonian et al. 2008).

A power law with photon index $\Gamma=2.65 \pm 0.19_{\text {stat }} \pm 0.20_{\text {sys }}$ and a differential normalisation at $1 \mathrm{TeV}$ of $\left(6.5 \pm 1.1_{\text {stat }} \pm\right.$ $\left.1.3_{\text {sys }}\right) \times 10^{-13} \mathrm{~cm}^{-2} \mathrm{~s}^{-1} \mathrm{TeV}^{-1}$ provides a satisfactory description of the data $\left(\chi^{2} /\right.$ d.o.f. $\left.=3.8 / 3\right)$ as shown in Fig. 2. The integral flux above $200 \mathrm{GeV}$ was found to be $\left(5.6 \pm 1.5_{\text {stat }} \pm 1.1_{\text {sys }}\right) \times$ $10^{-12} \mathrm{~cm}^{-2} \mathrm{~s}^{-1}$ and the energy flux in the range $0.5-5 \mathrm{TeV}$ is $\approx 2 \times 10^{-12} \mathrm{erg} \mathrm{cm}^{-2} \mathrm{~s}^{-1}$. 


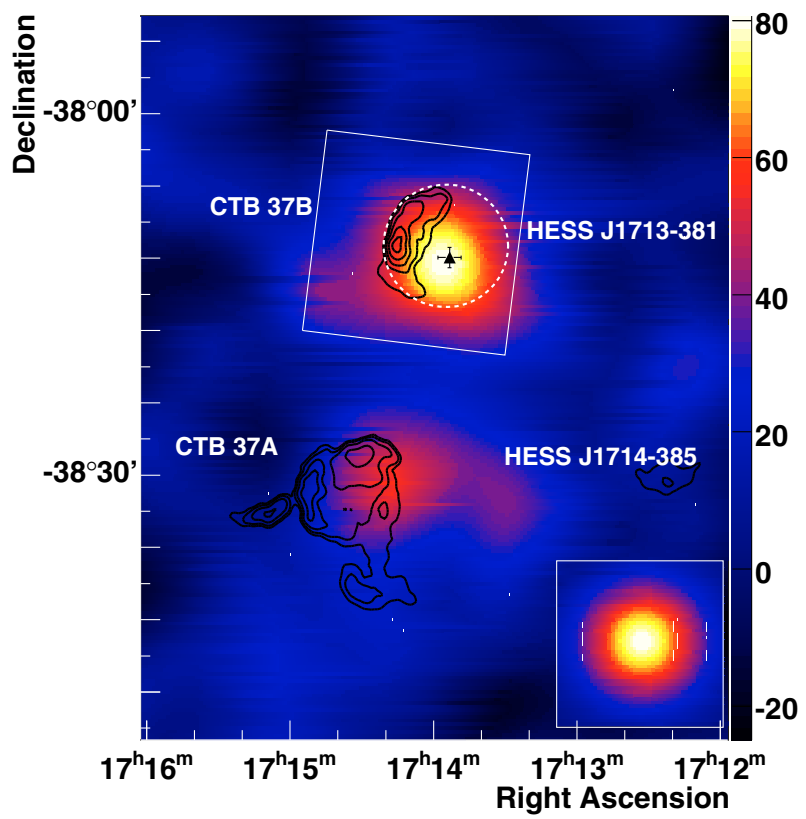

Fig. 1. Acceptance corrected $\gamma$-ray excess map of the SNR complex CTB 37 after Gaussian smoothing with a radius $\sigma_{\mathrm{sm}}$ of $2.9^{\prime}$. The black contours represent $0.08,0.16,0.32,0.64,1.28$ and $2.56 \mathrm{Jy} /$ beam radio emission from the Molonglo Galactic Plane Survey (beam size $\sim 1$; Green et al. 1999). The field of view of the Chandra observation is shown by a white box surrounding CTB 37B. The estimated size of the supernova remnant, centred on the position stated by Green (2006), is illustrated by a dashed white circle. The simulated PSF is shown in the box in the bottom right corner and is smoothed in the same way as the excess map. The colour scale of the map is in units of counts per $2 \pi \sigma_{\mathrm{sm}}^{2} \equiv$ counts per 0.0145 degrees $^{2}$.

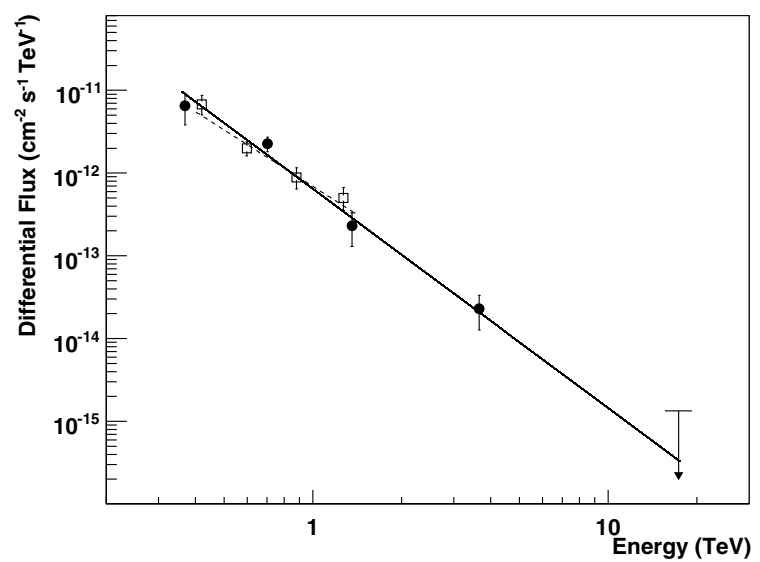

Fig. 2. Differential energy spectrum of HESS J1713-381. Solid symbols are derived from the full data set and are fitted with a power law with photon index $\Gamma=2.65 \pm 0.19$ (solid line). A 90\% confidence upper limit is given at energies above $10 \mathrm{TeV}$. The empty squares and the dashed line represent the data acquired during previous observations (Aharonian et al. 2006b).

A joint morphological fit of HESS J1714-385 and HESS J1713-381 was made with a model consisting of two Gaussian emission profiles convolved with the PSF of the instrument. The intrinsic rms width of HESS J1713-381 extracted from this fit, $2.6^{\prime} \pm 0.8^{\prime}$, is compatible with the size found during the HESS survey (Aharonian et al. 2006b). An unresolved thin shell of radius $r$ has an equivalent Gaussian rms of $\sigma \simeq 0.6 r$. For a filled sphere, $\sigma \simeq 0.45 r$. The measured

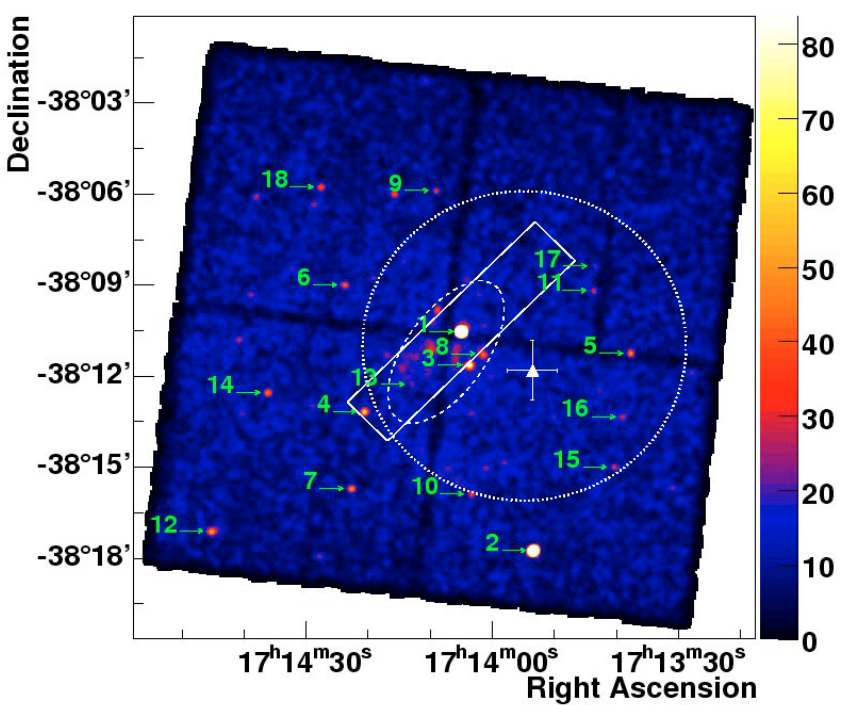

Fig. 3. X-ray image (1-5 keV) of the Chandra field of view showing the positions of the 18 detected point sources on the four ACIS CCD chips. The image is a count map after Gaussian smoothing with $\sigma_{\mathrm{sm}}=5^{\prime \prime}$. Source \#1 is located very close to one of the CCD chip edges. The position of the centroid of the TeV emission from HESS J1713-381 is marked by a white triangle. The white box shows the region from which the emission profile in Fig. 5 is taken and the dashed white ellipse shows the region from which the diffuse X-ray spectrum was extracted. The colour scale shows counts per $2 \pi \sigma_{\mathrm{sm}}^{2}$ and has been truncated to better show the fainter point sources in contrast to the background. The maximum prior to truncation was 1214 at the position of source \#1. This truncation forces the brighter sources to appear larger than their true size. As in Fig. 1, the dotted circle illustrates the estimated size of the SNR CTB 37B

width is consistent with a shell of radius $\sim 4-6^{\prime}$, compatible with the estimated radio shell size.

\section{X-ray observations and analysis}

The CTB 37B region was observed with the Advanced CCD Imaging Spectrometer (ACIS) of the Chandra X-ray Observatory on the 2nd February 2007 for $26 \mathrm{ks}$ (observation ID 6692). The raw data were analysed using the Chandra Interactive Analysis of Observations (CIAO version 3.4, CALDB version 3.4.1). The data set was unaffected by soft proton flares enabling the full observation time to be utilised for analysis purposes.

Figure 3 shows a count map of the region after Gaussian smoothing with a $5^{\prime \prime}$ radius to match the off-axis PSF. This image illustrates the positions of 18 point-like sources, described in Table 1, detected at a $>5 \sigma$ level within the Chandra field of view, using the CIAO wavdetect algorithm. Statistical errors on the positions are $\sim 3^{\prime \prime}$ for the weakest sources on each axis. The most significant of these sources, source \#1, is located $3^{\prime}$ northeast of the centroid of the TeV source HESS J1713-381.

Figure 4 shows an exposure corrected, smoothed excess map after removal of emission from the point sources described in Table 1. Extended diffuse emission is observed at a $>11 \sigma$ level to the east of HESS J1713-381 and appears to be contained within the region defined by the radio contours. The diffuse emission extends over all four ACIS CCD chips. This peak is spatially coincident with the radio shell, but is not aligned with the peak of the radio emission and is instead offset to the west by $\sim 1.6^{\prime}$. This shift is highly significant considering the $0.6^{\prime \prime}$ astrometric accuracy of Chandra. No evidence for significant temperature 
Table 1. Properties of all point sources detected above a $5 \sigma$ level in the Chandra field of view using the CIAO wavdetect algorithm.

\begin{tabular}{ccccrr}
\hline \hline ID & $\begin{array}{c}\text { Name } \\
\text { CXOU J }\end{array}$ & $\begin{array}{c}\text { RA } \\
17^{\mathrm{h}}\end{array}$ & $\begin{array}{c}\text { Dec } \\
-38^{\circ}\end{array}$ & Counts & $\begin{array}{r}\text { Significance } \\
\sigma\end{array}$ \\
\hline 1 & $171405.7-381031$ & $14^{\mathrm{m}} 5.758^{\mathrm{s}}$ & $10^{\prime} 31.32^{\prime \prime}$ & 1171 & 158.9 \\
2 & $171354.1-381844$ & $13^{\mathrm{m}} 54.129^{\mathrm{s}}$ & $18^{\prime} 44.24^{\prime \prime}$ & 445 & 38.6 \\
3 & $171404.3-381237$ & $14^{\mathrm{m}} 4.385^{\mathrm{s}}$ & $12^{\prime} 37.51^{\prime \prime}$ & 64 & 23.2 \\
4 & $171421.3-381310$ & $14^{\mathrm{m}} 21.38^{\mathrm{s}}$ & $13^{\prime} 10.19^{\prime \prime}$ & 55 & 19.6 \\
5 & $171438.4-381114$ & $14^{\mathrm{m}} 38.43^{\mathrm{s}}$ & $11^{\prime} 14.2^{\prime \prime}$ & 40 & 11.4 \\
6 & $171323.4-381641$ & $13^{\mathrm{m}} 23.45^{\mathrm{s}}$ & $16^{\prime} 41.8^{\prime \prime}$ & 40 & 9.8 \\
7 & $171424.4-380959$ & $14^{\mathrm{m}} 24.49^{\mathrm{s}}$ & $9^{\prime} 59.1^{\prime \prime}$ & 28 & 9.1 \\
8 & $171402.2-381115$ & $14^{\mathrm{m}} 2.224^{\mathrm{s}}$ & $11^{\prime} 15.4^{\prime \prime}$ & 19 & 8.4 \\
9 & $171409.7-380652$ & $14^{\mathrm{m}} 9.77^{\mathrm{s}}$ & $6^{\prime} 52.7^{\prime \prime}$ & 23 & 8.2 \\
10 & $171404.0-381652$ & $14^{\mathrm{m}} 4.09^{\mathrm{s}}$ & $16^{\prime} 52.4^{\prime \prime}$ & 23 & 7.6 \\
11 & $171444.3-380910$ & $14^{\mathrm{m}} 44.35^{\mathrm{s}}$ & $9^{\prime} 10.5^{\prime \prime}$ & 19 & 6.9 \\
12 & $171346.0-381705$ & $13^{\mathrm{m}} 46.08^{\mathrm{s}}$ & $17^{\prime} 5.8^{\prime \prime}$ & 37 & 6.5 \\
13 & $171413.6-381215$ & $14^{\mathrm{m}} 13.66^{\mathrm{s}}$ & $12^{\prime} 15.7^{\prime \prime}$ & 14 & 6.4 \\
14 & $171436.8-381331$ & $14^{\mathrm{m}} 36.88^{\mathrm{s}}$ & $13^{\prime} 31.8^{\prime \prime}$ & 31 & 6.3 \\
15 & $171341.0-381558$ & $13^{\mathrm{m}} 41.07^{\mathrm{s}}$ & $15^{\prime} 58.7^{\prime \prime}$ & 19 & 5.9 \\
16 & $171339.6-381320$ & $13^{\mathrm{m}} 39.61^{\mathrm{s}}$ & $13^{\prime} 20.0^{\prime \prime}$ & 15 & 5.4 \\
17 & $171344.2-380822$ & $13^{\mathrm{m}} 44.22^{\mathrm{s}}$ & $8^{\prime} 22.0^{\prime \prime}$ & 13 & 5.1 \\
18 & $171428.2-380545$ & $14^{\mathrm{m}} 28.27^{\mathrm{s}}$ & $5^{\prime} 45.6^{\prime \prime}$ & 23 & 5.1 \\
\hline
\end{tabular}

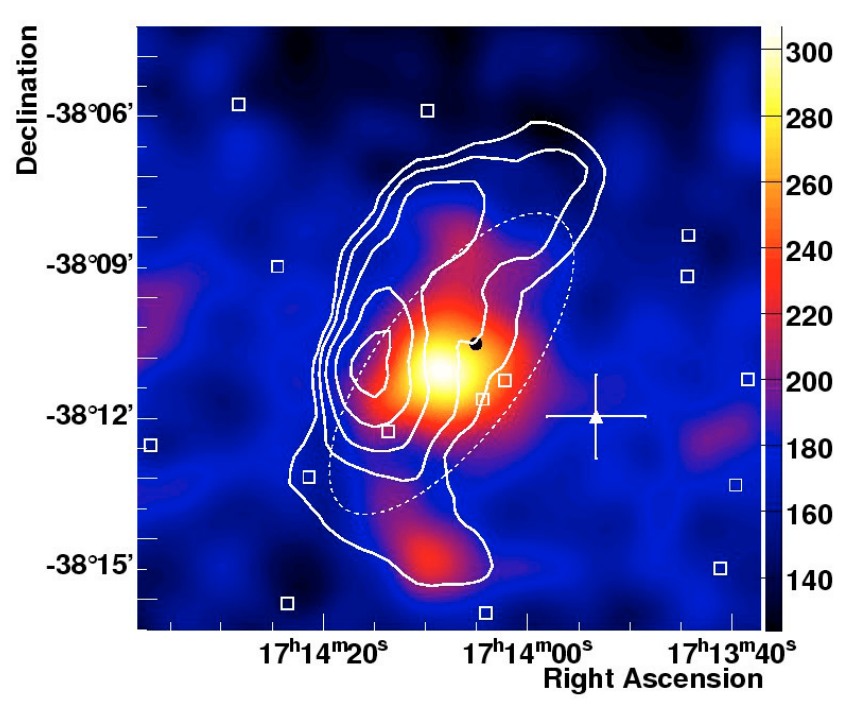

Fig. 4. Exposure corrected count map (1-5 keV) after subtraction of emission from the 18 point sources detected in the field of view. The image has been Gaussian smoothed with $\sigma_{\mathrm{sm}}=50^{\prime \prime}$ to highlight the diffuse emission. The colour scale is in units of counts per $2 \pi \sigma_{\mathrm{sm}}^{2}$. The black circle shows the position of source \#1. White square symbols show the location of the other point sources which have been removed from the image. The white contours represent the same radio contours as in Fig. 1. The position of the centroid of HESS J1713-381 is shown as a white triangle.

variations across the diffuse emission was found and spectral properties on each individual ACIS chip were consistent. Hence the region used to extract the reported diffuse spectrum extends over all four chips, and is indicated by a dashed white ellipse in Figs. 3 and 4.

Figure 5 shows a profile of the X-ray emission in the rectangular region shown in Fig. 3, after the removal of the 18 point sources found within the Chandra field of view. This region is centred on the position of the X-ray source CXOU J171405.7-381031 (source \#1). The simulated PSF model for source \#1 is shown in red. The rms width of the diffuse emission extracted from a Gaussian fit to the data (black curve, $\chi^{2} /$ d.o.f. $\left.=86.6 / 57\right)$ is $\sigma=1.2^{\prime} \pm 0.1^{\prime}$.

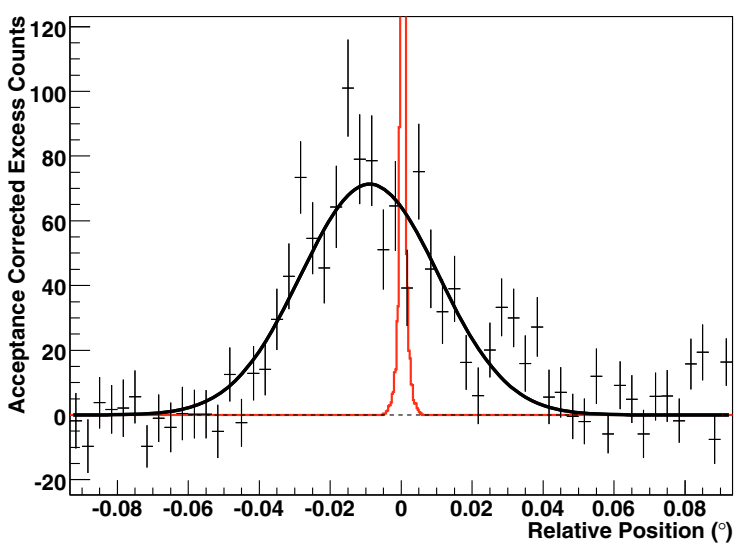

Fig. 5. Acceptance corrected emission profile taken from the rectangular region shown in Fig. 3, after the removal of emission from the 18 detected point sources. This region is centred on the best fit position of source \#1. A Gaussian fit to the data is shown by the smooth curve. The simulated PSF model for (the subtracted) source \#1 is shown in red. Source \#1 is $\sim 10$ times brighter ( 1200 counts) than the observed diffuse emission. The background is estimated from regions of equal size and shape displaced to the north and south of the diffuse emission.

The energy spectrum of source \#1 was taken from an integration region of radius $\sim 1.7^{\prime \prime}$ centred on the best fit position. This spectrum is well described by a single absorbed power law with $N_{\mathrm{H}}=\left(4.1_{-0.2}^{+0.3}\right) \times 10^{22} \mathrm{~cm}^{-2}$ and $\Gamma=3.3_{-0.1}^{+0.2}$ with $\chi^{2} /$ d.o.f. $=68.5 / 63$ (the probability of a blackbody model fit to this source was $\sim 0.2 \%$ ). Both sources \#2 and \#3 are consistent with blackbody models with $k T<0.5 \mathrm{keV}$. The other 15 point sources were too weak to allow a meaningful spectral analysis to be performed.

Regions around the 18 detected X-ray point sources were excluded from the data set before extracting the spectrum of the extended diffuse emission. The background was estimated from a large region covering all 4 ACIS chips and excluding regions around the point sources and the diffuse emission. The resulting spectrum was best fit with an absorbed nonequilibrium ionisation (NEI) model with $\tau=\left(7_{-3}^{+9}\right) \times 10^{10} \mathrm{~s} \mathrm{~cm}^{-3}$ $\left(\chi^{2} /\right.$ d.o.f. $\left.=68.9 / 62\right)$ and is shown in the bottom panel of Fig. 6 . 
Table 2. Best fit spectral parameters for the point source CXOU J171405.7-381031 and the diffuse X-ray emission with various models. Errors are given at the $1 \sigma$ level.

\begin{tabular}{|c|c|c|c|c|c|c|c|}
\hline \multirow[t]{2}{*}{ Element } & \multirow{2}{*}{$\begin{array}{c}\text { Model } \\
\text { (with absorption) }\end{array}$} & \multicolumn{4}{|c|}{ Best Fit Parameters } & \multirow{2}{*}{$\begin{array}{c}\text { Flux }(1-5 \mathrm{keV}) \\
\left(10^{-13} \mathrm{erg} \mathrm{cm}^{-2} \mathrm{~s}^{-1}\right)\end{array}$} & \multirow[t]{2}{*}{$\chi^{2} /$ d.o.f. } \\
\hline & & $N_{\mathrm{H}}\left(10^{22} \mathrm{~cm}^{-2}\right)$ & $\Gamma$ & $k T(\mathrm{keV})$ & $\tau\left(10^{10} \mathrm{~s} \mathrm{~cm}^{-3}\right)$ & & \\
\hline Source \#1 & Power law & $4.1_{-0.2}^{+0.3}$ & $3.3_{-0.1}^{+0.2}$ & & & 6.7 & $68.5 / 63$ \\
\hline Source \#1 & Blackbody & $2.1 \pm 0.2$ & & $0.76 \pm 0.03$ & & 5.9 & $100.2 / 63$ \\
\hline Source \#1 & $\begin{array}{c}\text { Two-temperature } \\
\text { Blackbody }\end{array}$ & $2.9_{-0.3}^{+0.2}$ & & $\begin{array}{c}0.52_{-0.04}^{+0.06} \\
1.6_{-0.3}^{+0.6}\end{array}$ & & 6.4 & $68.0 / 61$ \\
\hline Diffuse emission & NEI & $3.9_{-0.2}^{+0.3}$ & & $0.8 \pm 0.1$ & $7_{-3}^{+9}$ & 2.9 & $68.8 / 62$ \\
\hline Diffuse emission & NEI & $3.8_{-0.4}^{+0.5}$ & & $0.7 \pm 0.1$ & $5 \times 10^{3}$ (fixed) & 2.7 & $75.5 / 63$ \\
\hline Diffuse emission & Power law & $4.2_{-0.5}^{+0.6}$ & $5.3_{-0.5}^{+0.6}$ & & & 2.8 & $101.6 / 63$ \\
\hline
\end{tabular}

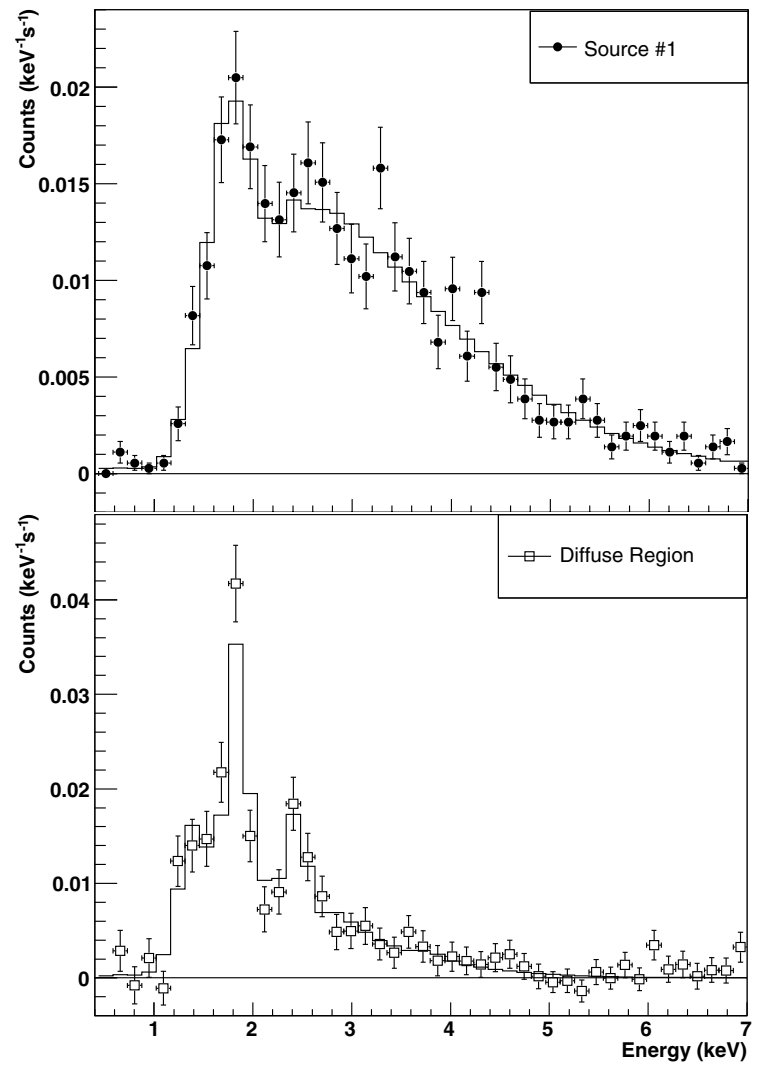

Fig. 6. Top: count rate versus energy for source \#1 (black filled symbols) compared to the best fit model for an absorbed power law. Bottom: count rate versus energy for the diffuse emission compared to the best fit model for an absorbed NEI model. Fit parameters are described in the text.

A marginally acceptable fit is also possible with $\tau$ set to the maximum allowed value of $5 \times 10^{13} \mathrm{~s} \mathrm{~cm}^{-3}$, indicating that the source is approaching equilibrium. No evidence was found for diffuse emission above $5 \mathrm{keV}$. Parameters of fits with different spectral models, including the absorbed fluxes, are given in Table 2. The consistent column densities from the model fits suggest a source \#1 and the diffuse emission lie at approximately the same distance.

Timing analysis of the source was difficult due to its position on the chip edge leading to an artificial modulation at $10^{-3} \mathrm{~Hz}$ caused by instrumental dither effects, and the $3.2 \mathrm{~s}$ frame time. However, we find no evidence for variability on $3 \times 10^{-3}$ to $0.2 \mathrm{~Hz}$ timescales.

\section{Discussion}

\subsection{The X-ray emission of the SNR CTB 37B}

The X-ray measurements presented here allow for a better assessment of the age and environment of CTB 37B than was previously possible. From the age and distance of the remnant an external density can be estimated. Unfortunately, the distance to the remnant is rather uncertain. Here we adopt a $7 \mathrm{kpc}$ distance based on the HI absorption measurements of Caswell et al. (1975) scaled to more recent galactic rotation measurements. Assuming that the thermal X-ray diffuse emission is indeed associated with the SNR and that electrons and ions are in thermal equilibrium, the temperature obtained from the NEI fit $(k T=0.8 \pm 0.1 \mathrm{keV})$ can be used to estimate the SNR age using the Sedov-Taylor phase equations (e.g., Borkowski et al. 2001). The temperature of the thermal emission implies a shock velocity, $v_{\mathrm{s}} \sim 800(T / 0.8 \mathrm{keV})^{1 / 2}\left(\mu / 0.62 m_{\mathrm{H}}\right)^{-1 / 2} \mathrm{~km} \mathrm{~s}^{-1}$ and the age of the remnant is estimated to be $\sim 4900(d / 7 \mathrm{kpc})\left(\mu / 0.62 m_{\mathrm{H}}\right)^{1 / 2}(T / 0.8 \mathrm{keV})^{-1 / 2}$ years (where $\mu$ is the mean mass per particle). At a distance of $7 \mathrm{kpc}$ the estimated radius of $5^{\prime}$ corresponds to an intrinsic size close to 10 pc. A spherical Sedov expansion then implies an external density of $\sim 0.8\left(E_{\mathrm{k}} / 10^{51} \mathrm{erg}\right) \mathrm{cm}^{-3}$ where $E_{\mathrm{k}}$ is the kinetic energy of the SN explosion. An age of 1600 years, compatible with an origin in the supernova of $\mathrm{AD} 393$, would imply $n \sim 0.1\left(E_{\mathrm{k}} / 10^{51} \mathrm{erg}\right) \mathrm{cm}^{-3}$.

There are three main complications that could affect these estimates. First, the Sedov solution holds only approximately, as the region surrounding CTB 37B appears to be inhomogeneous, leading to an asymmetric expansion. Second, the temperature equilibrium between electrons and ions assumed above is not seen in many young SNRs, see Rakowski (2005) for a recent review. In the case of non-equilibration, the velocity estimate must be increased by a factor $\sqrt{\left(1+T_{\mathrm{i}} / T_{\mathrm{e}}\right) / 2}$ (asuming a pure hydrogen gas), making the remnant younger by the same factor. Finally, efficient acceleration of cosmic rays at the shock would result in less heating of the thermal gas for a given shock velocity (because a fraction of the dissipated kinetic energy goes into the non-thermal population). The more efficient the acceleration, the faster the shock must be to achieve a given gas temperature, leading to an over estimate of the SNR age using the conventional Sedov-Taylor gas dynamic solution. In the approximation of a steady-state, plane-parallel shock (see, e.g., Drury \& Voelk 1981), it may be shown that if the fraction of the downstream enthalpy in accelerated particles is $\vartheta$, then the velocity estimate must be increased by a factor of approximately $1 / \sqrt{1-\vartheta}$, and the age estimate decreased accordingly. For example, the combined effect of non-equilibrium temperatures, $T_{\mathrm{i}}=3 T_{\mathrm{e}}$, and very 
efficient CR acceleration, $\vartheta=0.4$, would change the estimated age of the remnant to $\sim 2700$ years.

The thermal X-ray flux can be used as a cross-check for this scenario. Due to absorption the luminosity implied by the measured flux is only a small fraction of the radiated power. The de-absorbed bolometric flux, $f_{\text {bol }}$, is $\approx 2.6 \times 10^{-11} \mathrm{erg} \mathrm{cm}^{-2} \mathrm{~s}^{-1}$. At $0.8 \mathrm{keV}\left(\sim 10^{7} \mathrm{~K}\right)$, the cooling coefficient, $\Lambda$ is $\approx 2.7 \times$ $10^{-23} \mathrm{erg} \mathrm{cm}^{3} \mathrm{~s}^{-1}$ (Sutherland \& Dopita 1993). The ion density in the emission region is connected to $f_{\text {bol }}$ via $f_{\text {bol }}=$ $\Lambda V n^{2} / 4 \pi d^{2}$, assuming thermal equilibrium and equal number densities for electrons and ions. Taking the volume, $V$, to be that of an ellipsoid with principle axes equal to the FWHM of the X-ray emision along its major axis, $a=2.8^{\prime}$ (as shown in Fig. 5); the FWHM in the perpendicular direction, $b=1.9^{\prime}$; and the FWHM along the line of sight (estimated as $f \times a$ ); we find $n \sim 2(f d / 7 \mathrm{kpc})^{-1 / 2} \mathrm{~cm}^{-3}$. The non-observation of thermal X-ray emission in other parts of the shell implies $n \lesssim 1 \mathrm{~cm}^{-3}$ in these regions. If this emission occurs in a shock compressed region the external density is likely a factor $\sim 4-7$ lower and hence broadly consistent with the previous estimate. A general picture therefore emerges of CTB $37 \mathrm{~B}$ as a few 1000-year-old SNR expanding into a (somewhat inhomogeneous) medium with average density $n \sim 0.5 \mathrm{~cm}^{-3}$. This discussion assumes that the bulk of the thermal X-ray emission originates in the shocked surrounding medium; if a significant fraction were to originate in shocked SN ejecta, the above estimates would have to be substantially revised.

\subsection{X-ray point sources}

None of the 18 detected X-ray point sources were found to be spatially coincident with the centroid of the observed VHE emission and there is no obvious candidate for a compact central object of the SNR CTB 37B. For the three brightest sources (\#1, \#2, and \#3), there are sufficient statistics for spectral analysis to be attempted and all are apparently pointlike in nature. Source \#1 (CXOU J171405.7-381031) is by far the brightest and lies in coincidence with the radio shell of CTB 37B and close to the maximum of the diffuse X-ray emission of the shell. As it has a measured $N_{\mathrm{H}}$ consistent with that of the SNR shell, it seems plausible that there is a physical association of these two objects. In this context, source \#1 may represent the X-ray emission of an isolated neutron star. We find no clear counterpart for this object at optical/infrared wavelengths; the closest catalogued source is the star GSC 2.3 S8PL008678 from the Guide Star Catalogue (version 2.3.2) (Space Telescope Science Institute \& Osservatorio Astronomico di Torino 2007), offset by $\sim 4^{\prime \prime}$. The closest $8 \mu \mathrm{m}$ GLIMPSE (Benjamin et al. 2003) source apparent by visual inspection lies $\sim 3^{\prime \prime}$ away.

Whilst the luminosity of source \#1 $\left(\sim 4 \times 10^{33} \mathrm{erg} \mathrm{s}^{-1}\right)$ lies in a range fairly typical for the X-ray nebulae of Vela-like PWN (for example PSR J1823-13 and PSR J1811-1925, see Kargaltsev et al. 2007), the lack of extended, non-thermal emission around source \#1 argues strongly against the PWN hypothesis. In addition, an identification of source \#1 with the neutron star from the SN explosion of CTB 37B would imply a large proper motion of this object. Assuming a birth place of the pulsar at the geometric centre of the remnant $\left(R A=17^{\mathrm{h}} 13^{\mathrm{m}} 55^{\mathrm{s}}\right.$, Dec $=-38^{\circ} 11^{\prime} 0^{\prime \prime}$; Green 2006) and an age $t$ yields an implied "kick" velocity of $\sim 1000(d / 7 \mathrm{kpc})(t / 5000 \text { years })^{-1} \mathrm{~km} \mathrm{~s}^{-1}$. This estimate lies towards the upper edge of the range of velocities observed for pulsars (10- 2000 $\mathrm{km} \mathrm{s}^{-1}$; Iben \& Tutukov 1996;
Chatterjee \& Cordes 2002; Arzoumanian et al. 2002) ${ }^{1}$. A pulsar with such a velocity is likely to be associated with a bowshock morphology pulsar wind nebula, as seen for example in G 359.23-0.82 ("the Mouse"; Gaensler et al. 2004) where the pulsar has a velocity of $\approx 600 \mathrm{~km} \mathrm{~s}^{-1}$. No such feature is detected in this case.

Source \#2 is spatially coincident with the star USNO-A2.00450-26795004 and has a soft thermal spectrum $(k T \sim 0.2 \mathrm{keV})$ and relatively low $N_{\mathrm{H}}$ of $<6 \times 10^{21} \mathrm{~cm}^{-2}$, consistent with emission from a nearby star. Source \#3 also has a low temperature $(\sim 0.4 \mathrm{keV})$ black-body spectrum but the closest catalogued star is $\sim 3^{\prime \prime}$ away. This source is spatially coincident with the diffuse X-ray emission, and has a consistent column density $\left((6 \pm 2) \times 10^{22} \mathrm{~cm}^{-2}\right)$ suggesting that these objects may be physically associated. The closest source to the centroid of VHE emission is source \#8 but the 19 excess counts are not sufficient for spectral analysis. This source has no catalogued stellar counterpart.

\subsection{The nature of the $\gamma$-ray source HESS J1713-381}

The position and size of HESS J1713-381 make an association with CTB 37B very likely. However, as in the case of other objects such as HESS J1813-178 (Funk et al. 2007a), there is possible ambiguity between an origin of the $\mathrm{TeV}$ emission in the SNR shell and a pulsar wind nebula interpretation. Here we discuss these alternative explanations in turn.

\subsubsection{TeV emission from a PWN}

There are now $>10$ associations of young pulsars and $\mathrm{TeV}$ $\gamma$-ray sources. These associations suggest a typical fraction of $\sim 1 \%$ for the conversion of rotational energy into $\mathrm{TeV}$ emission (Carrigan et al. 2007). Assuming that the explosion of CTB 37B left behind a pulsar, the TeV emission could be explained as inverse Compton emission from "relic" electrons accelerated soon after the pulsar birth. Assuming IC scattering only on the cosmic microwave background (CMB), the synchrotron cooling time of electrons responsible for $\mathrm{TeV}$ emission is $\sim 8000(B / 10 \mu \mathrm{G})^{-2}\left(E_{\gamma} / 1 \mathrm{TeV}\right)^{-1 / 2}$ years, long enough that electrons injected immediately after the pulsar birth would continue to contribute to the $\mathrm{TeV}$ emission. In contrast, $\sim 3 \mathrm{keV}$ synchrotron emission is associated with higher energy $\left(\sim 80(B / 10 \mu \mathrm{G})^{-1 / 2} \mathrm{TeV}\right)$ electrons which cool somewhat faster: $t_{\text {sync }} \approx 1500(B / 10 \mu \mathrm{G})^{-3 / 2}\left(\epsilon_{\text {sync }} / 3 \mathrm{keV}\right)^{-1 / 2}$ years, and would hence be confined to a region close to the present pulsar position. The prototype of the class of $\mathrm{TeV}$ bright PWN, HESS J1825-137 (Aharonian et al. 2006c), is associated with the $\sim 2 \times 10^{4}$ year old, high-spindown luminosity $\left(\dot{E} \approx 3 \times 10^{36} \mathrm{erg} \mathrm{s}^{-1}\right)$ pulsar PSR B1823-13. The TeV source HESS J1713-381 has similar spectral shape $(\Gamma=2.65 \pm 0.19$ versus $\Gamma \approx 2.4$ ), but is apparently somewhat smaller (diameter $10 \mathrm{pc}$ cf. $40 \mathrm{pc}$ ), younger (5 kyr cf. $20 \mathrm{kyrs})$ and dimmer $(0.5-5 \mathrm{TeV}$ luminosity $\sim 1.2 \times 10^{34} \mathrm{erg} \mathrm{s}^{-1} \mathrm{cf} . \sim 8 \times 10^{34} \mathrm{erg} \mathrm{s}^{-1}$ ).

However, even if CXOU J171405.7-381031 is indeed a high kick velocity pulsar, the absence of extended non-thermal X-ray emission is very difficult to explain in this scenario. An explanation of HESS J1713-381 by inverse Compton emission from

\footnotetext{
1 We note that both the $\mathrm{X}$-ray and radio emission suggest a denser medium on the eastern side of the remnant implying that the explosion centre may have been significantly further east than the current geometrical centre, hence reducing the required proper motion of the pulsar.
} 
ultra relativistic electrons in the wake of the pulsar therefore seems rather implausible.

\subsubsection{TeV emission from the SNR shell}

Whilst there is a clear offset of the centroid of HESS J1713-381 from the thermal X-ray and radio emission of the shell, the TeV centroid is consistent with the estimated geometrical centre of the remnant. In addition, the observed size of the $\mathrm{TeV}$ source is consistent with emission in a thin shell of radius $\sim 4.5^{\prime}$, consistent with the radius of the radio shell. If the observed radio and $\mathrm{X}$-ray emission from the eastern part of the shell is due to a local density and/or magnetic field enhancement, then it seems plausible that particle acceleration may also be taking place in the western part. In a scenario where the $\mathrm{TeV}$ emission originates from IC scattering of $>\mathrm{TeV}$ electrons, the emission is expected to directly trace the distribution of such electrons (assuming uniform radiation fields on the scale of the SNR). However, X-ray synchrotron emission is expected from the same population of electrons with a flux $\sim F_{\mathrm{TeV}}(B / 3 \mu \mathrm{G})^{2} /\left(U_{\mathrm{rad}} / U_{\mathrm{CMB}}\right)$, assuming that there is no cut-off in the electron energy spectrum between the range probed by the TeV IC emission and that probed by 2-10 keV synchrotron emission (see above). The lack of measured non-thermal X-ray emission in the shell implies an X-ray synchrotron flux $F_{\text {sync }}$ significantly lower than the observed thermal flux of $\sim 3 \times 10^{-13} \mathrm{erg} \mathrm{cm}^{-2} \mathrm{~s}^{-1}$. Given the measured TeV flux of $2 \times 10^{-12} \mathrm{erg} \mathrm{cm}^{-2} \mathrm{~s}^{-1}(0.5-5 \mathrm{TeV})$, this IC scenario implies either an implausibly low B-field of $\sim 1 \mu \mathrm{G}$ or a rather sharp cut-off in the electron spectrum around $\sim 40 \mathrm{TeV}$.

A more natural explanation may be an origin of the $\mathrm{TeV}$ emission via the decay of neutral pions produced in protonproton interactions in the region of the SNR shell. Following Aharonian et al. (1994), the expected $\gamma$-ray flux is $F(>1 \mathrm{TeV}) \sim$ $10^{-12} \theta\left(E_{\mathrm{k}} / 10^{51} \mathrm{erg}\right)(d / 7 \mathrm{kpc})^{-2}\left(n / 0.5 \mathrm{~cm}^{-3}\right) \mathrm{cm}^{-2} \mathrm{~s}^{-1}$ where $\theta$ is the $\mathrm{CR}$ acceleration efficiency. The measured flux above $1 \mathrm{TeV}$ $(3.9 \pm 0.7) \times 10^{-13} \mathrm{~cm}^{-2} \mathrm{~s}^{-1}$ implies efficient CR acceleration. The relatively soft measured $\mathrm{TeV}$ spectrum $(\Gamma=2.65 \pm 0.19)$ is somewhat surprising in this scenario, but could be interpreted as the consequence of a cut-off in the proton spectrum at energies somewhat lower than seen, for example, in RX J1713.7-3946 $\left(E_{\max } \sim 100 \mathrm{TeV}\right.$, Aharonian et al. 2007).

\subsubsection{Association with the EGRET source 3EG J1714-3857}

The source HESS J1713-381 lies close to the $99 \%$ confidence contour of the $>100 \mathrm{MeV}$ source 3EG J1714-3857 (Hartman et al. 1999) and so this object could be considered as a potential counterpart to the observed $\mathrm{TeV}$ excess. The SNR RX J1713.7-3946 has also been suggested as a counterpart to this emission (Butt et al. 2002), as has the newly discovered $\gamma$-ray source HESS J1714-385 (Aharonian et al. 2008). Despite exhibiting much weaker TeV emission than RX J1713.7-3946, HESS J1713-381 may be considered as a viable counterpart to 3EGJ1714-3857 due to its softer spectral shape $(\Gamma \approx$ 2.7). An extrapolation of the $\mathrm{TeV}$ spectrum down to $\mathrm{GeV}$ energies is marginally consistent with the measured flux of 3EG J1714-3857. However, such a spectral match is not unlikely for a chance pairing of HESS and EGRET sources, given the respective instrumental sensitivities and typical spectral shapes (Funk et al. 2007b). The angular resolution and flux sensitivity of GLAST (Thompson 2004) should allow this issue to be resolved in the near future.

\section{Summary}

The new X-ray and $\gamma$-ray data presented here represent a major improvement in our knowledge of the CTB 37B/HESS J1713-381 system. The discovery of thermal $\mathrm{X}$-ray emission from the shell allows the uncertainties on the age and ambient density of the remnant to be considerably reduced, with likely values of $\sim 5000$ years and $\sim 0.5 \mathrm{~cm}^{-3}$ emerging. The non-thermal point-source CXOU J171405.7-381031, which is detected embedded in the diffuse thermal emission, may be a neutron star associated with CTB 37B, but considerable difficulties exist with this interpretation. It seems likely that the $\mathrm{TeV}$ emission originates in the shell of CTB 37B. Whilst an $\mathrm{IC}$ origin of the $\mathrm{TeV}$ emission cannot be excluded, the decay of neutral pions produced in proton-proton interactions in the (entire) shell of the supernova remnant seems to be the most natural explanation.

Acknowledgements. The support of the Namibian authorities and of the University of Namibia facilitating the construction and operation of HESS is gratefully acknowledged, as is the support by the German Ministry for Education and Research (BMBF), the Max Planck Society, the French Ministry for Research, the CNRS-IN2P3 and the Astroparticle Interdisciplinary Programme of the CNRS, the UK Science and Technology Facilities Council (STFC), the IPNP of the Charles University, the Polish Ministry of Science and Higher Education, the South African Department of Science and Technology and National Research Foundation, and by the University of Namibia. We appreciate the excellent work of the technical support staff in Berlin, Durham, Hamburg, Heidelberg, Palaiseau, Paris, Saclay, and in Namibia in the construction and operation of the equipment. We would like to thank Ryoko Nakamura for helpful discussions regarding the $\mathrm{X}$-ray analysis.

\section{References}

Aharonian, F., et al. (HESS Collaboration) 2005, A\&A, 437, L7 Aharonian, F., et al. (HESS Collaboration) 2006a, A\&A, 457, 899 Aharonian, F., et al. (HESS Collaboration) 2006b, ApJ, 636, 777 Aharonian, F., et al. (HESS Collaboration) 2006c, A\&A, 460, 365 Aharonian, F., et al. (HESS Collaboration) 2007, A\&A, 464, 235 Aharonian, F., et al. (HESS Collaboration) 2008, [arXiv: 0803.0702] Aharonian, F. A., Drury, L. O., \& Voelk, H. J. 1994, A\&A, 285, 645 Arzoumanian, Z., Chernoff, D. F., \& Cordes, J. M. 2002, ApJ, 568, 289 Benjamin, R. A., Churchwell, E., Babler, B. L., et al. 2003, PASP, 115, 953 Berge, D., Funk, S., \& Hinton, J. 2007, A\&A, 466, 1219

Borkowski, K. J., Lyerly, W. J., \& Reynolds, S. P. 2001, ApJ, 548, 820 Brand, J., \& Blitz, L. 1993, A\&A, 275, 67

Butt, Y. M., Torres, D. F., Romero, G. E., Dame, T. M., \& Combi, J. A. 2002, Nature, 418, 499

Carrigan, S., Hinton, J. A., Hofmann, W., et al. 2007, [arXiv: 0709.4094]

Caswell, J. L., Murray, J. D., Roger, R. S., Cole, D. J., \& Cooke, D. J. 1975, A\&A, 45, 239

Chatterjee, S., \& Cordes, J. M. 2002, ApJ, 575, 407

Clark, D. H., \& Stephenson, F. R. 1975, The Observatory, 95, 190

Downes, A. 1984, MNRAS, 210, 845

Drury, L. O., \& Voelk, J. H. 1981, ApJ, 248, 344

Frail, D. A., Goss, W. M., Reynoso, E. M., et al. 1996, AJ, 111, 1651

Funk, S., Hinton, J. A., Moriguchi, Y., et al. 2007a, A\&A, 470, 249

Funk, S., Reimer, O., Torres, D. F., \& Hinton, J. A. 2007b, [arXiv: 0710. 1584]

Gaensler, B. M., \& Slane, P. O. 2006, ARA\&A, 44, 17

Gaensler, B. M., van der Swaluw, E., Camilo, F., et al. 2004, ApJ, 616, 383

Green, A. J., Cram, L. E., Large, M. I., \& Ye, T. 1999, ApJS, 122, 207

Green, D. A. 2006, A Catalogue of Galactic Supernova Remnants, 2006 April Version, Astrophysics Group, Cavendish Laboratory, Cambridge, UK, http: //www . mrao.cam.ac.uk/surveys/snrs/

Hartman, R. C., Bertsch, D. L., Bloom, S. D., et al. 1999, ApJS, 123, 79

Hinton, J. 2007, [arXiv: 712.3352]

Iben, I. J., \& Tutukov, A. V. 1996, ApJ, 456, 738

Kargaltsev, O., Pavlov, G. G., \& Garmire, G. P. 2007, ApJ, 660, 1413

Kassim, N. E., Weiler, K. W., \& Baum, S. A. 1991, ApJ, 374, 212

Li, T.-P., \& Ma, Y.-Q. 1983, ApJ, 272, 317

de Naurois, M. 2006, [arXiv:0607247]

Rakowski, C. E. 2005, Adv. Space Res., 35, 1017

Reynoso, E. M., \& Mangum, J. G. 2000, ApJ, 545, 874 
Schmidt, M., \& Blaauw, A. 1965, Galactic structure (Chicago, ILL, USA: University of Chicago Press)

Space Telescope Science Institute, \& Osservatorio Astronomico di Torino 2007, VizieR Online Data Catalog, 1305, 0

Sutherland, R. S., \& Dopita, M. A. 1993, ApJS, 88, 253

Thompson, D. J. 2004, New Astron. Rev., 48, 543

1 Max-Planck-Institut für Kernphysik, PO Box 103980, 69029

Heidelberg, Germany

2 Yerevan Physics Institute, 2 Alikhanian Brothers St., 375036

Yerevan, Armenia

3 Centre d'Étude Spatiale des Rayonnements, CNRS/UPS, 9 Av. du

Colonel Roche, BP 4346, 31029 Toulouse Cedex 4, France

4 Universität Hamburg, Institut für Experimentalphysik, Luruper

Chaussee 149, 22761 Hamburg, Germany

5 Institut für Physik, Humboldt-Universität zu Berlin, Newtonstr. 15, 12489 Berlin, Germany

6 LUTH, Observatoire de Paris, CNRS, Université Paris Diderot, 5 Place Jules Janssen, 92190 Meudon, France

7 IRFU/DSM/CEA, CE Saclay, 91191 Gif-sur-Yvette Cedex, France

8 University of Durham, Department of Physics, South Road, Durham DH1 3LE, UK

9 Unit for Space Physics, North-West University, Potchefstroom 2520, South Africa

10 Laboratoire Leprince-Ringuet, Ecole Polytechnique, CNRS/IN2P3, 91128 Palaiseau, France

11 Laboratoire d'Annecy-le-Vieux de Physique des Particules, CNRS/IN2P3, 9 Chemin de Bellevue, BP 110, 74941 Annecy-leVieux Cedex, France

12 Astroparticule et Cosmologie (APC), CNRS, Université Paris 7 Denis Diderot, 10, rue Alice Domon et Leonie Duquet, 75205 Paris Cedex 13, FranceUMR 7164 (CNRS, Université Paris VII, CEA, Observatoire de Paris)

13 Dublin Institute for Advanced Studies, 5 Merrion Square, Dublin 2, Ireland

14 Landessternwarte, Universität Heidelberg, Königstuhl, 69117 Heidelberg, Germany
15 Laboratoire de Physique Théorique et Astroparticules, CNRS/IN2P3, Université Montpellier II, CC 70, Place Eugène Bataillon, 34095 Montpellier Cedex 5, France

16 Universität Erlangen-Nürnberg, Physikalisches Institut, ErwinRommel-Str. 1, 91058 Erlangen, Germany

17 Laboratoire d'Astrophysique de Grenoble, INSU/CNRS, Université Joseph Fourier, BP 53, 38041 Grenoble Cedex 9, France

18 Institut für Astronomie und Astrophysik, Universität Tübingen, Sand 1, 72076 Tübingen, Germany

19 LPNHE, Université Pierre et Marie Curie Paris 6, Université Denis Diderot Paris 7, CNRS/IN2P3, 4 Place Jussieu, 75252 Paris Cedex 5, France

20 Institute of Particle and Nuclear Physics, Charles University, V Holesovickach 2, 18000 Prague 8, Czech Republic

21 Institut für Theoretische Physik, Lehrstuhl IV: Weltraum und Astrophysik, Ruhr-Universität Bochum, 44780 Bochum, Germany

22 University of Namibia, Private Bag 13301, Windhoek, Namibia

23 Obserwatorium Astronomiczne, Uniwersytet Jagielloński, Kraków, Poland

24 Nicolaus Copernicus Astronomical Centre, Warsaw, Poland

25 School of Physics and Astronomy, University of Leeds, Leeds LS2 9JT, UK

e-mail: phy3j2ls@ast.leeds.ac.uk

26 School of Chemistry and Physics, University of Adelaide, Adelaide 5005, Australia

27 Toruń Centre for Astronomy, Nicolaus Copernicus University, Toruń, Poland

28 European Associated Laboratory for Gamma-Ray Astronomy, jointly supported by CNRS and MPG

29 Stanford University, HEPL \& KIPAC, Stanford, CA 94305-4085, USA

30 Kavli Institute for Particle Astrophysics, SLAC, 2575 Sand Hill Road, Menlo Park, CA 94025, USA

31 Université Bordeaux 1; CNRS/IN2P3; Centre d'Études Nucléaires de Bordeaux Gradignan, UMR 5797, Chemin du Solarium, BP 120, 33175 Gradignan, France 